\title{
A level set formulation for the numerical simulation of impact of surge fronts
}

\author{
A SALIH ${ }^{1}$ and $\mathrm{S}$ GHOSH MOULIC ${ }^{2}$ \\ ${ }^{1}$ Department of Mechanical Engineering, National Institute of Technology, \\ Tiruchirappalli 620015, India \\ ${ }^{2}$ Department of Mechanical Engineering, Indian Institute of Technology, \\ Kharagpur 721302, India \\ e-mail: salih@nitt.edu
}

MS received 18 November 2005; revised 3 April 2006

\begin{abstract}
In this paper we present a level set-based algorithm for the solution of incompressible two-phase flow problems. The technique is applied to the numerical simulation of impact of two surge fronts resulting from the collapse of liquid columns. The incompressible Navier-Stokes equations are solved using a projection method based on forward Euler time-stepping. The Hamilton-Jacobi type equation for the transport of level set function is carried out by a high resolution fifth-order accurate WENO scheme. For efficient implementation of the WENO scheme we have proposed grid staggering for the level set function. The solution of the pressure Poisson equation is obtained using an efficient preconditioned conjugate gradient method. It is shown that the present formulation works very well for large density and viscosity ratios. For the purpose of validation, we have simulated small-amplitude free sloshing of liquid in a container and the wellknown two-dimensional broken-dam problem of Martin and Moyce. Simulations of impact of surge fronts have been carried out and the results are discussed.
\end{abstract}

Keywords. Free surface flows; level set methods; impact of surge fronts.

\section{Introduction}

Flows involving more than one phase arise in a multitude of engineering applications. A physical understanding of multiphase flows offers problems of far greater complexity than encountered with single-phase flows. A primary reason for this is that the location of fluid interface is not known a priori. Hence any solution procedure for computing such flows must include a method for locating and advancing the interface as well as for treating the interface boundary conditions for velocity and pressure. Over the years, a number of computational methods have been developed for solving flow with moving interfaces. Direct numerical simulations of multiphase flow, where the full continuum equations are solved on a computational grid sufficiently fine to resolve all continuum scales, date back to the origin of computational fluid dynamics itself. The oldest and still the most popular approach to compute multi-fluid and 
multiphase flows is to embed the front directly on a regular, stationary grid. The marker-andcell (MAC) method (Harlow \& Welch 1965), where marker particles are used to identify each fluid, and the volume-of-fluid (VOF) method (Hirt \& Nicholas 1981), where a marker function is used, are well-known examples. Traditionally, the main difficulty in using these methods has been the maintenance of a sharp boundary between the different fluids and computation of the surface tension. A critical and comprehensive review of methods for computing flow with moving interfaces has been carried out by Floryan \& Rasmussen (1989). A recent review of the widely used VOF method can be found in Scardovelli \& Zaleski (1999).

A more recent addition to the collection of methods that capture fluid interfaces on a fixed grid is the level set method of Osher \& Sethian (1988). Here the interface is modelled as the zero level set of a smooth phase function (level set function), $\phi$, defined over the whole computational domain. The level set function is advected with the background flow field, thus propagating the interface exactly as the zero level set of $\phi$. Since the interface is captured implicitly, this algorithm is capable of capturing the intrinsic geometric properties of highly complicated interfaces in quite a natural way. Further, it automatically takes care of topological changes in the interface such as merging and breakups. Since their introduction, level set methods have seen tremendous expansion in applications in diverse areas like twophase flows, combustion, crystal growth, image processing, computer vision, etching, and grid generation, to name just a few. One of the first papers that describes the application of level set method to incompressible flows is by Sussman et al (1994). They present results for several two-dimensional cases involving the motion of air bubbles in water and falling water drops in air. Later, Chang et al (1996) give detailed derivations of level set based governing equations of motion for incompressible flows. The level set method is reviewed by Osher \& Fedkiw (2001) and by Sethian \& Smereka (2003). Also. the monograph by Sethian (1999) on level set methods serves as an excellent reference material. In the present paper, we have used the level set method for simulation of impact of two surge fronts and consequent jet formation.

\section{Problem definition}

The present problem of impact of two surge fronts is a modification of the classical twodimensional broken dam problem without tail water. The initial setup consists of two identical liquid columns placed at the opposite sides of the container. The instantaneous release of the retaining wall of the liquid columns results in two identical surge fronts moving in opposite directions. These surge fronts collide at the centre of the container to form a water jet which rises enough to hit the top wall of the container and then falls down under the action of gravity. We have taken up this problem because of the rich physics involved in it, as it has all the ingredients of a difficult free surface problem because of the complex topological changes associated with the interface motion. During the evolution of the interface, it develops sharp corners, merging and breakups take place, as well as interactions with the solid no-slip boundary. We simulate the entire sequence of events using our level set formulation. The specific geometry employed in the simulation is illustrated in figure 1. Two square liquid columns with width $L=1$ are enclosed at opposite sides of a container $4.5 \mathrm{~L}$ units long and $1.5 \mathrm{~L}$ units high. Reynolds number Re, and Froude number Fr are the relevant dimensionless parameters of the present problem. The length scale is chosen to be the width of the liquid column $L$. We define the characteristic velocity $U=(g L)^{1 / 2}$ and the characteristic time $t=(L / g)^{1 / 2}$. With these definitions, we have $\operatorname{Re}=\left(\rho^{2} g L^{3} / \mu^{2}\right)^{1 / 2}$ and $\mathrm{Fr}=1$ where $\rho$ and $\mu$ are the density and viscosity of liquid respectively and $g$ is the gravitational acceleration. 


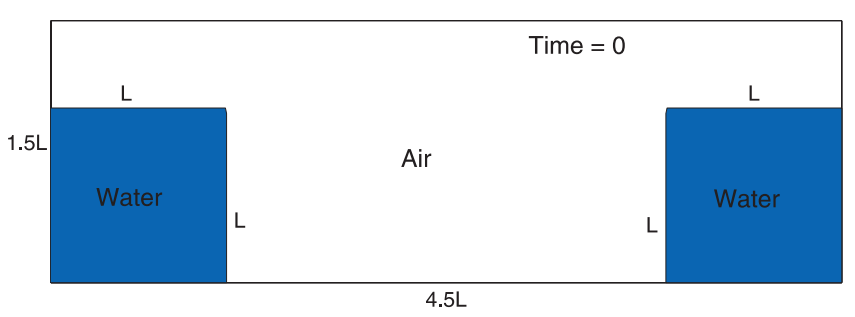

Figure 1. Initial setup for study of the impact of two travelling surge fronts.

\section{Governing equation and numerical method}

To study the impact of two surge fronts, we consider the laminar flow of an incompressible, Newtonian viscous fluid with variable fluid properties. The governing equations in nondimensional form are described as

$$
\begin{aligned}
\nabla \cdot \mathbf{u} & =0 \\
\frac{\partial \mathbf{u}}{\partial t}+(\mathbf{u} \cdot \nabla) \mathbf{u} & =\frac{1}{\rho}\left[-\nabla p+\frac{1}{\operatorname{Re}} \nabla \cdot\left[\mu\left(\nabla \mathbf{u}+(\nabla \mathbf{u})^{\mathrm{T}}\right)\right]\right]-\frac{1}{\operatorname{Fr}} \mathbf{e}_{y},
\end{aligned}
$$

where $t$ is the time, $\mathbf{u}=\langle u, v\rangle$ the velocity, $p$ the pressure, $\rho$ and $\mu$ are the fluid density and viscosity respectively, and $\mathbf{e}_{y}$ is the unit vector along the vertical $y$-axis. Note that all these variables are dimensionless quantities. Equation (1) represents the mass conservation and (2) represents the balance of momentum. For the present work we have developed an explicit Navier-Stokes solver on a staggered grid (Salih 2007) as described in Peyret \& Taylor (1983). Though the projection algorithm by Peyret \& Taylor (1983) is based on finite difference formulation, we have adapted it to the finite volume formulation for present study. The temporal discretization has been done using forward Euler time stepping. The convection terms are treated by a second-order upwind scheme and the viscous terms by standard central differencing schemes. The numerical solution of the pressure Poisson equation resulting from the projection step is obtained using an efficient preconditioned conjugate gradient method (Golub \& Loan 1996).

\section{Level set method}

The general idea behind the level set method is to embed the hypersurface (representing the moving interface) in a domain of one-higher dimension than the dimension of the hypersurface. Consider a bounded domain $\Omega$ with boundary $\partial \Omega$, containing two immiscible fluids. Both fluids divide the domain into two subdomains $\Omega^{-}$and $\Omega^{+}$by the fluid-fluid interface $\Gamma$. The level set function $\phi$ is chosen in such a way that it assumes negative values in region $\Omega^{-}$and positive values in region $\Omega^{+}$, so that the fluid interface $\Gamma(t)$ corresponds to zero level set of $\phi$. Mathematically, the interface $\Gamma(t)$ is given by

$$
\Gamma(t)=\{\mathbf{x} \in \Omega: \phi(\mathbf{x}, t)=0\} .
$$

The level set evolution for a velocity field $\mathbf{u}(\mathbf{x}, t)$ is governed by

$$
\partial \phi / \partial t+\mathbf{u} \cdot \nabla \phi=0, \quad \phi(\mathbf{x}, 0)=\phi_{0}(\mathbf{x})
$$


where $\phi_{0}$ embeds the initial location of the interface. The above Hamilton-Jacobi type equation accurately moves the zero level set according to the velocity field $\mathbf{u}(\mathbf{x}, t)$ even through merging and breaking of the fluid interfaces. The numerical discretization of level set equation (4) is carried out by the high resolution fifth-order accurate WENO scheme introduced by Jiang \& Peng (2000). For efficient implementation of the WENO scheme we have used grid staggering for the level set function. We have located $\phi$ at the vertices of the main control volumes. This kind of grid staggering avoids nonuniform grids adjacent to the computational boundary and consequently no special treatment of WENO stencil is required near the boundary. In order to avoid numerical instabilities while solving (2), density and viscosity are smeared out and modelled as smooth functions of $\phi$ by assuming that the interface has a finite thickness $\varepsilon$. The smoothed density and viscosity are now given by

$$
\begin{aligned}
& \rho_{\varepsilon}(\phi)=\rho_{-}+\left(\rho_{+}-\rho_{-}\right) H_{\varepsilon}(\phi), \\
& \mu_{\varepsilon}(\phi)=\mu_{-}+\left(\mu_{+}-\mu_{-}\right) H_{\varepsilon}(\phi), \quad \text { and } \\
& H_{\varepsilon}(\phi)= \begin{cases}0, & \text { if } \phi<-\varepsilon ; \\
\frac{\phi+\varepsilon}{2 \varepsilon}+\frac{1}{2 \pi} \sin \left(\frac{\pi \phi}{\varepsilon}\right), & \text { if }|\phi| \leq \varepsilon ; \\
1, & \text { if } \phi>\varepsilon .\end{cases}
\end{aligned}
$$

where $\rho_{-}, \rho_{+}, \mu_{-}$, and $\mu_{+}$are density and viscosity of fluid in regions $\Omega^{-}$and $\Omega^{+}$respectively. $H_{\varepsilon}(\phi)$ is the regularized Heaviside function. The interface thickness parameter $\varepsilon$ is typically taken as $1.5 h$, where $h$ is the grid size. This results in having an interface thickness of three grid cells when $\phi$ is a distance function. It is shown that the present formulation works very well for large density and viscosity ratios. As with any interface capturing schemes the level set method is also plagued by loss of mass. However, we demonstrate that the mass loss is not a major issue for the present problem.

It may be noted that though the level set function, $\phi$, is initialized as signed normal distance from the interface, under the evolution of the advection equation (4), it does not necessarily remain so. This problem is overcome by reinitializing the level set function periodically (Sussman et al 1994).

\section{Results and discussion}

In this section we present relevant results of our computations. First, we validate the method by considering a standard benchmark problem. Subsequently, the results for two-dimensional broken dam problem and the impact of surge fronts problem are presented.

\subsection{Free sloshing of liquid in a container}

The free-surface algorithm and the code have been tested by simulating the small-amplitude free sloshing of liquid in a two-dimensional rectangular container. Since analytical solutions exist for this problem, many researchers routinely used this problem for validating the multiphase Navier-Stokes algorithms. The problem consists of the gravity-induced oscillation of an interface without any external excitation of the container. The problem setup is as follows. Consider a partially filled two-dimensional rectangular container. The free surface of the initially quiescent fluid is given a sinusoidal displacement. Under action of gravity, the free surface begins to oscillates as a standing wave. For sufficiently small amplitudes of oscillation, 
the linearized potential theory (Stoker 1992) gives the natural frequency of oscillation of the $m$ th mode, in dimensionless form, as

$$
\omega_{m}=\left[\frac{k_{m}}{\operatorname{Fr}} \tanh \left(k_{m} H\right)\right]^{1 / 2}
$$

where $H$ is the height of the unperturbed free surface, Fr the Froude number, and $k_{m}(=2 \pi / \lambda)$ the wave number of the perturbation, with $\lambda$ the wavelength. Also, the time-dependent free surface height at a location $x$, in dimensionless form, is given by

$$
z=H+A \cos \left(k_{m} x\right) \sin \left(\omega_{m} t\right)
$$

where $A$ is a parameter whose value corresponds to the maximum amplitude of the wave at $x=0$ (left wall of the container). The dimensionless $x$ - and $y$-components of velocity in the liquid respectively are given by the relations,

$$
\begin{aligned}
u & =-a k_{m} \sin \left(k_{m} x\right) \cosh \left(k_{m} y\right) \cos \left(\omega_{m} t\right) \quad \text { and } \\
v & =a k_{m} \cos \left(k_{m} x\right) \sinh \left(k_{m} y\right) \cos \left(\omega_{m} t\right),
\end{aligned}
$$

where the parameter $a$ is related to the amplitude parameter $A$ by the equation

$$
a=A / \operatorname{Fr} \omega_{m} \cosh \left(k_{m} H\right) .
$$

It is to be noted that these results were derived by ignoring the effect of the fluid above (outer fluid) the liquid which is being sloshed. However, since the level set method treats the liquid free surface as the interface between two fluids, it is necessary to consider the outer fluid also while modelling the sloshing problem. To facilitate direct quantitative comparison between numerical and analytical results, we could set the density and viscosity of the outer fluid to an arbitrarily low value. In our computation, we have selected the properties of water for the liquid which is being sloshed and the properties of air for the outer fluid.

The Froude number Fr and the Reynolds number Re are the relevant dimensionless parameters of the present problem. The definition of these numbers are given in $\S 2$. The length scale, in this case, is chosen to be the breadth of the tank $L$. The following input data were used for the simulation:

- The square tank is taken as of width $L=1$ and height $H_{\text {tank }}=1$,

- Density and viscosity ratios of the two fluids are taken as 820 and 55.5 respectively: these ratios correspond to that of water and air, the gravitational acceleration $g=9.81$,

- The unperturbed height of the free surface $H=0.5$, which gives a fill fraction (= volume of liquid/total volume of tank) of 0.5 ,

- The no-slip boundary condition is applied on all the walls,

- The Navier slip condition is implemented where the interface touches the walls,

- The Reynolds number $\mathrm{Re}=3132092$, and the Froude number Fr $=1$,

- The initial shape is cosinusoidal with wavelength taken to be twice the width of the tank. The corresponding wave number is then equal to $\pi$. The amplitude of the wave $A$ is taken as $0.002 H$, to ensure that the nonlinear effects are indeed negligible and a legitimate comparison is possible with the linear theory,

- The computational domain is discretized by a $64 \times 64$ uniform control volume mesh, and the time step is taken equal to the grid size, which gives the dimensionless time step, $\Delta t=0.005$ and satisfies the necessary CFL condition for stability, 

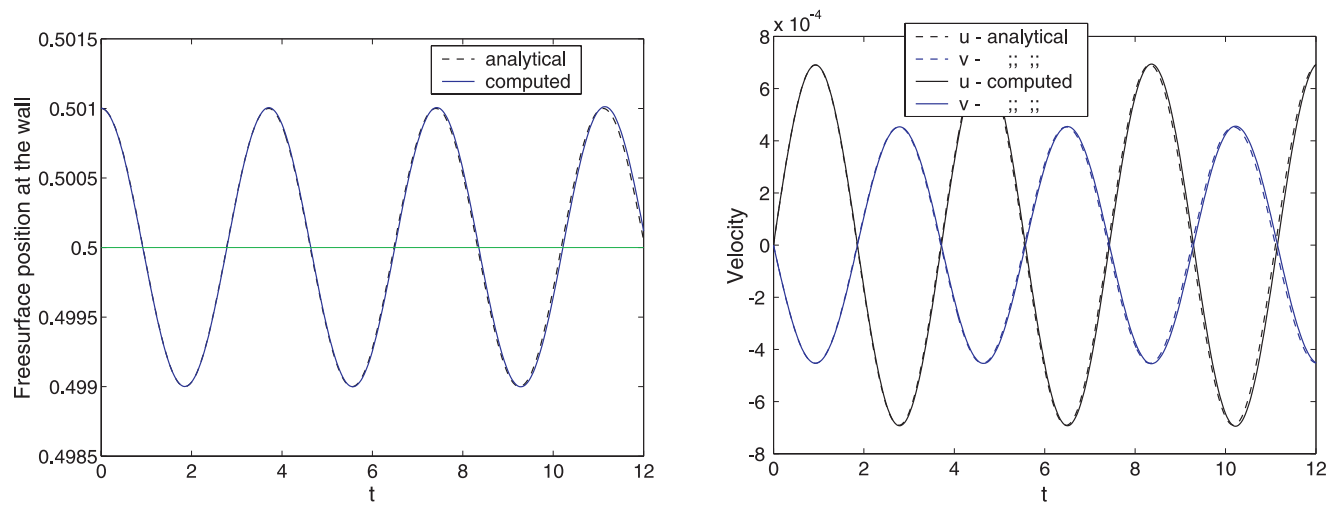

Figure 2. Time evolution of free surface height at the left wall of the container (left), the horizontal line indicates the unperturbed free surface; transient behaviour of velocity at a typical point in the liquid (right).

- The calculations are allowed to proceed until a dimensionless time, $t=12$, that requires 768 number of time steps.

The extremely high value of Reynolds number indicates that the viscous effects are indeed negligible and amplitude essentially remains the same over several time periods. This is confirmed by our results. The left frame in figure 2 shows the time evolution of the free surface height at the left wall. The analytical and the computed results are almost indistinguishable. The right frame in the same figure displays the time evolution of velocity components at a typical point inside the liquid domain. Again, the computed results lie right on top of the analytical results. The left and right frames in figure 3 respectively display the transient behaviour of the kinetic energy of the liquid and the normalized volume of liquid. It appears that the maximum kinetic energy of the liquid slightly increases towards the end of the computation. The reason for this unphysical behaviour can be attributed to the numerical error and the consequent volume gain by the liquid towards the end of computation as shown
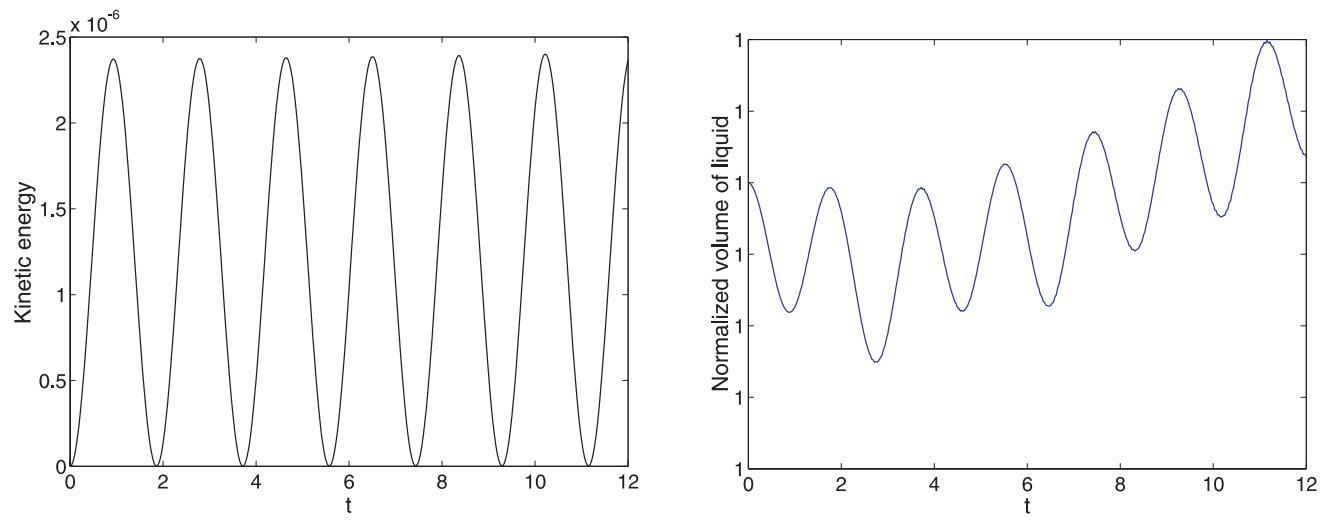

Figure 3. Transient behaviour of kinetic energy of the liquid (left) and normalized volume of liquid (right). 


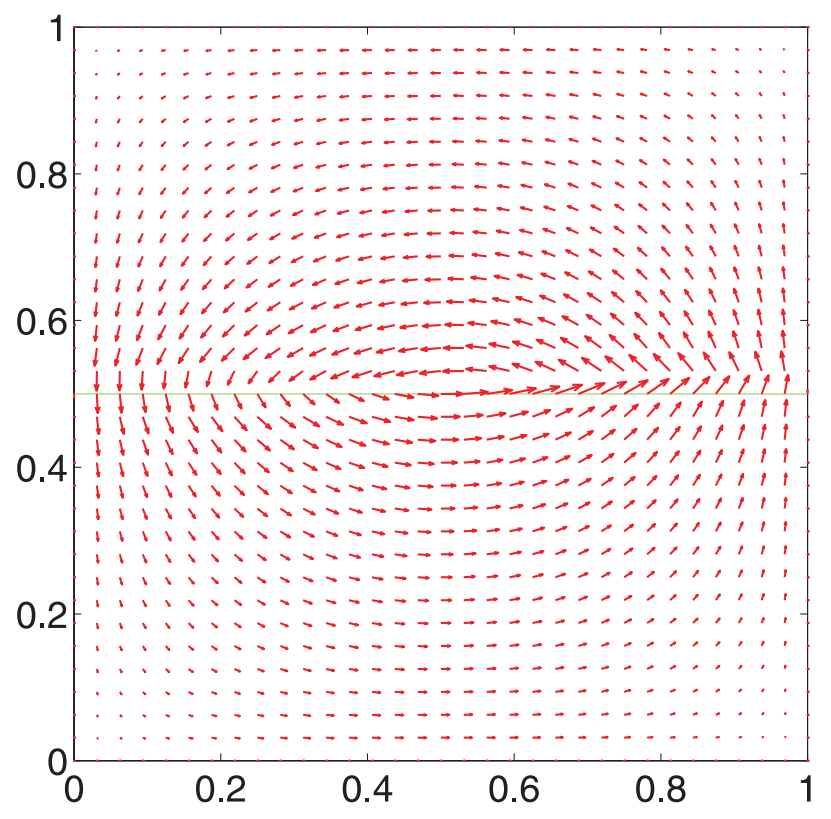

Figure 4. Velocity field at the end of computation.

in the figure. This volume gain is estimated to be $0.0000179 \%$ of the initial volume of the liquid which is indeed a very small quantity. To facilitate a quantitative comparison theoretical result, we estimate the period of oscillation on $32^{2}$ and $64^{2}$ grids. The theoretical period of oscillation $\left(=2 \pi / \omega_{n}\right)$ calculated using (6) is 3.7015 . The computed periods on $32^{2}$ and $64^{2}$ grids respectively are 3.7365 and 3.7143 . Thus, we find that the oscillation period is $0.945 \%$ longer than the theoretical period on a $32^{2}$ grid and $0.346 \%$ longer than on a $64^{2}$ grid. These results show that the level set formulation correctly captures the physics of the problem and performs well even in the presence of large density and viscosity ratios. Finally, we display the velocity field at the end of the computation in figure 4 .

\subsection{Two-dimensional broken dam problem}

We begin by considering the simulation of the well-known two-dimensional broken dam problem and compare the results with the experimental data of Martin \& Moyce (1952). The importance in solving the broken dam problem numerically lies in the fact that there are many common features between this problem and the impact of surge fronts. Unlike the sloshing considered in the previous section, the broken dam problem and impact of surge front problem involve large distortions of the interface. The Reynolds number for the simulation is taken as $\mathrm{Re}=42792$ to corresponds to that of the Martin and Moyce experimental conditions. The computations are performed on three sets of grids, $48 \times 16,96 \times 32$, and $144 \times 48$, to establish the grid's independence. The dimensionless time step $\Delta t=0.005,0.0018,0 \cdot 0009$ are chosen for the coarsest to finest grids. The calculations are allowed to proceed until the surge front reaches the right wall of the container. Figure 5 presents the free surface profiles at various times with time interval 0.4 . The predicted time history of the liquid front moving along the dry bed and the column height position are displayed in figure 6 along with the experimental results of Martin \& Moyce (1952). It can be observed that the plot 


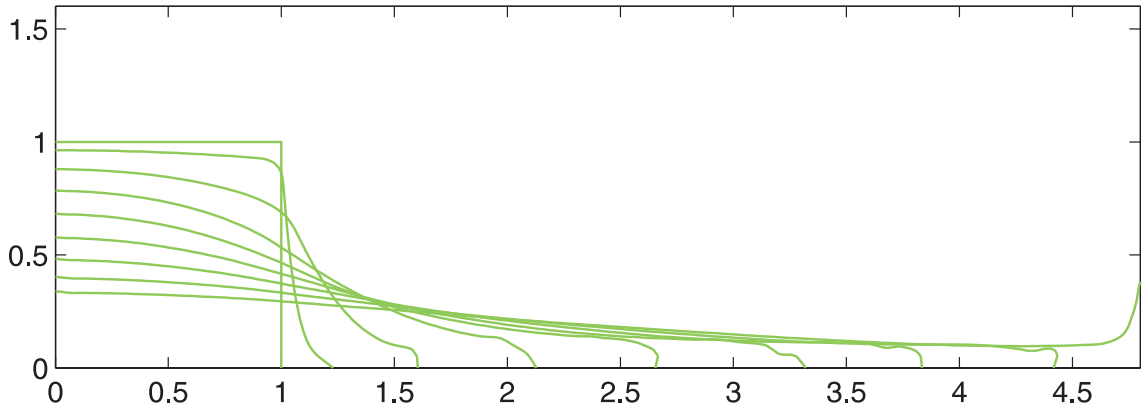

Figure 5. Transient free surface profile for 2-D broken dam problem.

for the numerical wave front position agrees well with the data of Martin \& Moyce (1952). However, the numerical simulation seems to be slightly ahead of experimental data. We believe that this shift can be attributed to the small difference in the initial condition between simulation and experiment. The removal of the retaining wall in the real world experiment cannot be instantaneous, whereas in the numerical simulation it is. This accounts for the time-gap between the simulation and the experiment. It can also be observed that the plot for the numerical wave front position shows a slight wavy pattern. It is not clear whether the wavy pattern observed is physically correct or is due to some effects not accounted for by the numerical model. The plot for column height agrees exceptionally well with the data of Martin \& Moyce (1952). This is remarkable considering the uncertainties in the experimental data and the approximations employed in the numerical model, specifically the way we have implemented the Navier slip condition where the interface touches the wall (Salih 2007).

\subsection{Impact of surge fronts}

The Reynolds number for the simulation of the impact of surge fronts is taken as 21396. In this case, we adopt a variable time step strategy for the following reason. We have observed that during the impact process the local velocity of the background fluid (air) becomes large necessitating a smaller value of $\Delta t$ to satisfy the $C F L$ number requirements. Using this small
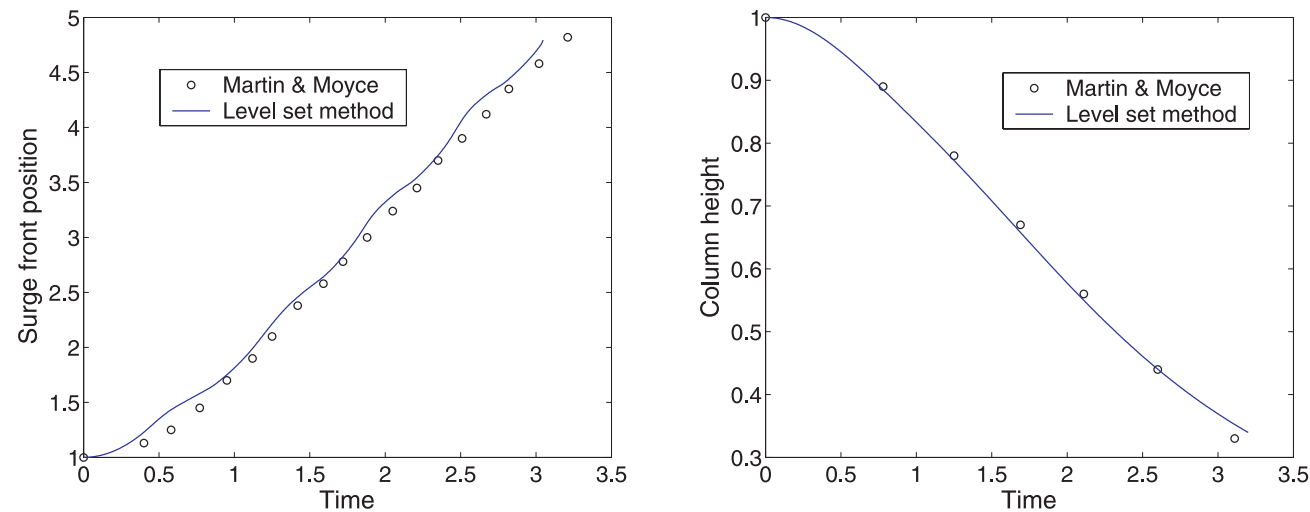

Figure 6. Transient surge front position (left) and transient column height (right). 
value of $\Delta t$ throughout the entire simulation period would increase the computation time to almost double. This is especially undesirable on finer grids. A trial run of this simulation on a coarse grid of $49 \times 16$ grid would provide us the necessary information about the time of impact. This information is used in selecting the variable time step on the finer grid of $145 \times 48$. The computations are advanced until a dimensionless time $t=6 \cdot 0$. We could not get a converged result with a dimensionless time step $\Delta t=0.001$ on a $145 \times 48$ grid. We have run the problem again with a variable time step of $\Delta t=0.001$ up to a time of 1.2 , $\Delta t=0.00066$ between $t=1.2$ and 1.6 , and again $\Delta t$ is reset to 0.001 and calculations continued until $t=6 \cdot 0$. The number of time steps required for the entire process is 6606 as opposed to 9091 when $\Delta t=0.00066$ were used.

The results of our numerical computations on a $145 \times 48$ grid is displayed in figure 8 which shows the free surface profiles at various times with time interval 0.4. It can be seen that the impact of the surge fronts results in the formation of a symmetric jet which rises up to the top wall of the container. As the jet rises, its speed gradually reduces and thickness increases. By the time it reaches the top wall the fluid velocity has reduced to a low value and hence there is no splashing action seen when the jet hits the top wall. Under the action of gravity the jet loses all its velocity and transforms itself to a liquid column which starts to collapse. During this process the formation of the neck near the top wall can be clearly seen. The neck moves downwards and gets progressively thinner and finally pinch-off takes place about $t=4.4$. The detached liquid column while collapsing turns into two opposite moving waves with a tongue-like formation at the tips. The folding of the tongue results in entrainment of air in the liquid. The computations are stopped just before the waves start hitting the side walls of the container.

Figure 7 shows the variation of normalized liquid volume with time. From this plot it can be seen that the nature of variation of liquid volume during the computation does not follow any specific pattern. In fact, it depends on the grid size used for computation. This is because the numerical diffusion tends to smooth out the high curvature region which might have developed during the evolution of the interface. Thus during a given time period, the volume may be lost in some part of the domain and gained in some other part depending upon the local sign of curvature of the interface. We note that at the end of computation, there is a loss in the net volume of liquid on a $49 \times 16$ grid $(11.3 \%)$ and a $97 \times 32$ grid $(12.4 \%)$

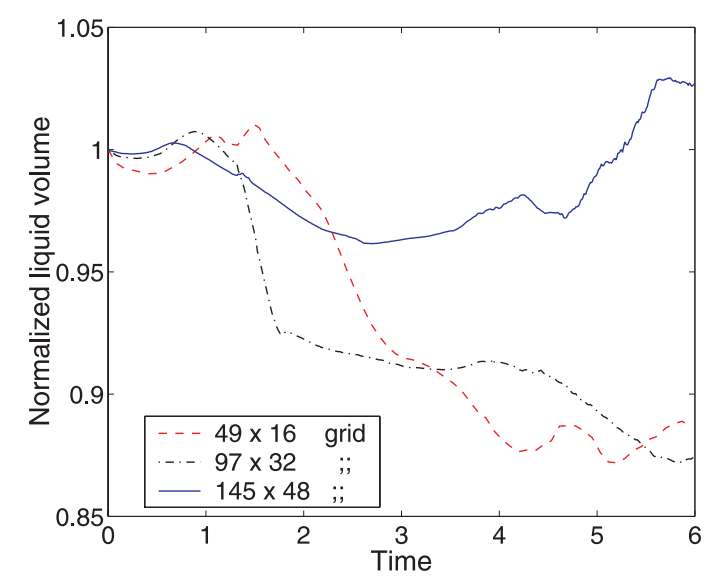

Figure 7. Time history of normalized liquid volume for impact of surge problem. 


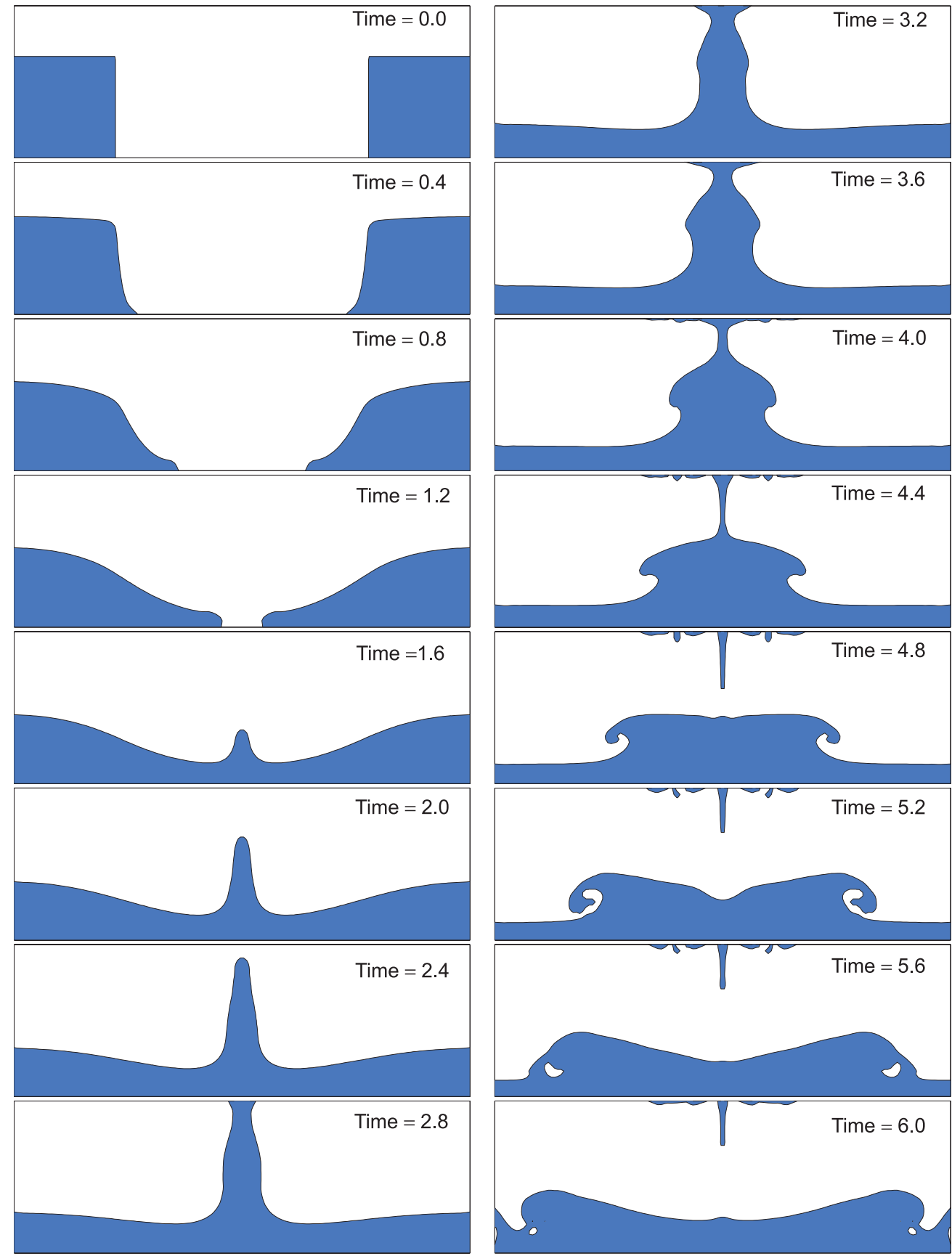

Figure 8. Transient evolution of free surface profiles for impact of travelling surge fronts.

but a gain in net volume on a $145 \times 48$ grid by $2.77 \%$. We remark here that the solution on the $145 \times 48$ grid is not rigourously tested for grid independence. Further resolution of free surface profile may be obtained on still finer grids. With our limited computational resources 
the computational time become prohibitively expensive on finer grids. Also, as there is no related previous work either numerical or experimental available, we are unable to compare our results. As a final remark, we mention that surface tension has been neglected in the computation. Surface tension can play an major role in the later stages of simulation when small-scale phenomena are important.

\section{Conclusion}

We solved the small-amplitude free sloshing in a partially filled rectangular container for validating the code and the methodology. The predicted results are in excellent agreement with theoretical results. We have successfully used the level set method to simulate the entire process of collapsing liquid columns and their impacts. It clearly demonstrate that the level set method is an elegant numerical tool when complex topological changes of free-surface is involved in the fluid dynamics problems. Although the level set method does not inherently conserve the individual fluid volumes, it is encouraging to see that the long time calculation (as we have done for the present simulation) does not incur excessive volume loss if sufficiently finer grids are used.

\section{References}

Chang Y C, Hou T Y, Merriman B, Osher S 1996 A level set Formulation of eulerian interface capturing methods for incompressible fluid flows. J. Comput. Phys. 124: 449-464

Floryan J M, Rasmussen H 1989 Numerical methods for viscous flows with moving boundaries. Appl. Mech. Rev. 42: 323-340

Golub G, Van Loan C 1996 Matrix computations 3rd edn (Baltimore: The Johns Hopkins University Press)

Jiang G-S, Peng D 2000 Weighted ENO schemes for Hamilton-Jacobi equations. SIAM J. Sci. Comput. 21: 2126-2143

Harlow F H, Welch J E 1965 Numerical study of large-amplitude free-surface motions. Phys. Fluids 8: 2182-2189

Hirt C W, Nichols B D 1981 Volume of fluid (VOF) methods for dynamics of free boundaries. J. Comput. Phys. 39: 201-225

Martin J C, Moyce W J 1952 An experimental study of the collapse of liquid columns on a rigid horizontal plane. Philos. Trans. R. Soc. A224: 312-324

Osher S, Fedkiw R 2001 Level set methods: An overview and some recent results, J. Comput. Phys. 169: 463-502

Osher S, Sethian J A 1988 Fronts propagating with curvature-dependent speed: Algorithm based on Hamilton-Jacobi formulations. J. Comput. Phys. 79: 12-49

Peyret R, Taylor T D 1983 Computational methods for fluid flow (Springer-Verlag, New York)

Salih A 2007 Numerical simulation of two-fluid flows with sharp interfaces using level set method $\mathrm{PhD}$ thesis, Indian Institute of Technology, Kharagpur

Scardovelli R, Zaleski S 1999 Direct numerical simulation of free-surface and interfacial flow. Annu. Rev. Fluid Mech. 31: 567-603

Sethian J A 1999 Level set methods and fast marching methods (Cambridge: University Press)

Sethian J A, Smereka P 2003 Level set methods for fluid interfaces. Annu. Rev. Fluid Mech. 35: 341372

Stoker J J 1992 Water waves: The mathematical theory with applications 2nd edn (New York: Wiley Interscience)

Sussman M, Smereka P, Osher S 1994 A level set approach for computing solutions to incompressible two-phase flow. J. Comput. Phys. 114: 146-159 